\title{
Subclavian steal syndrome decreases neurogenesis in the cerebellar cortex and affects cognitive function in rabbits
}

\author{
XIAO-YANG FU ${ }^{1,2}$, ZHI-DONG ZHANG ${ }^{1,2}$, KAI LIANG ${ }^{1,2}$, SHUAI-TAO SHI ${ }^{1,2}$, GUO-QUAN WANG ${ }^{1,2}$, \\ KE-WEI ZHANG ${ }^{1,2}$, KUN LI ${ }^{1,2}$, WEI-XIAO LI ${ }^{1,2}$, TIAN-XIAO LI ${ }^{1,2}$ and SHUI-TING ZHAI ${ }^{1,2}$ \\ ${ }^{1}$ Department of Vascular and Endovascular Surgery, Henan Provincial Hospital; \\ ${ }^{2}$ Department of Vascular and Endovascular Surgery, The Affiliated People's Hospital, \\ Zhengzhou University Medical School, Zhengzhou, Henan 450003, P.R. China
}

Received September 1, 2014; Accepted July 7, 2015

DOI: $10.3892 /$ etm.2015.2670

\begin{abstract}
Subclavian steal syndrome (SSS) is a condition characterized by a steno-occlusive impairment of the proximal subclavian artery. The majority of patients with SSS are asymptomatic, while symptomatic patients present with neurological symptoms. SSS is a risk factor for cerebral ischemia, which reacts badly upon cognitive function; however, it remains unknown whether SSS is able to cause progressive cognitive impairment. In the present study, the potential effects of SSS on cognitive function were investigated using atherosclerotic rabbits as a model of SSS. A total of 48 male New Zealand rabbits were divided into the control, sham and SSS groups. The results of eyeblink experiments indicated no significant differences among the three groups; however, SSS did appear to exert a negative impact on neurogenesis in the cerebellar cortex. In order to further clarify the mechanisms underlying this SSS-mediated reduction in cell proliferation, the energy metabolism, immune function and oxidative stress statuses were evaluated by determining the levels of adenosine triphosphate (ATP), adenosine, interleukin (IL)-1 $\beta$, IL-6, malondialdehyde, 8-hydroxy-2'-deoxyguanosine, CuZn-superoxide dismutase and catalase. The results showed that the levels of extracellular ATP in the cerebellar cortex had decreased, while levels of adenosine had also decreased. These findings suggest that SSS is able to inhibit neurogenesis in the cerebellar cortex by decreasing the extracellular ATP levels. Furthermore, these changes may result in an impairment of the cognition of the rabbits. The early diagnosis and treatment of SSS may, therefore, prevent or mitigate cognitive impairment in the future.
\end{abstract}

Correspondence to: Dr Shui-Ting Zhai, Department of Vascular and Endovascular Surgery, Henan Provincial Hospital, 7 Weiwu Road, Zhengzhou, Henan 450003, P.R. China

E-mail: zhaishuitingzz@yeah.net

Key words: subclavian steal syndrome, neurogenesis, cognition, adenosine triphosphate, cerebellar cortex

\section{Introduction}

Subclavian steal syndrome (SSS) is a condition characterized by a steno-occlusive impairment of the proximal subclavian artery (SA). SSS is most frequently observed in Caucasians aged $>50$ years, due to the increased incidence of atherosclerosis in this population. SSS causes reversal flow in the ipsilateral vertebral artery (VA) away from the brainstem, resulting in vertebrobasilar insufficiency (1). The primary cause of SSS is atherosclerosis. The majority of patients with SSS are asymptomatic, while symptomatic patients present with neurological symptoms, including light-headedness, ataxia, vertigo, dizziness, hearing loss, confusion, headache, nystagmus, visual disturbances, focal seizures and presyncope (2-4).

Treatment of SSS remains controversial (5). Since the majority of patients with SSS are asymptomatic, there has been debate as to the necessity of conducting early treatment. Alcocer et al (6) recommended that patients presenting with low symptomatology or non-life threatening symptoms be treated with a conventional approach; however, Sharma et al (7) suggested that SSS-induced chronic brainstem hypoperfusion could lead to a gradual slowing of speech and gait in patients and that early diagnosis and treatment could result in an improved quality of life. Notably, SSS is a risk factor for cerebral ischemia, which may negatively affect cognitive function (8); however, it remains unknown whether SSS is able to cause progressive cognitive impairment. Thus, the aim of the present study was to investigate the potential effects of SSS on cognitive function in an atherosclerotic rabbit model of SSS (9).

\section{Materials and methods}

Establishment of the SSS model in atherosclerotic rabbits. All experimental procedures involving animals were performed in accordance with the guidelines from the National Institutes of Health and approved by the Animal Care and Use Committee of Zhengzhou University Medical School (Zhengzhou, China). A study population of 48 male New Zealand rabbits (mean body weight, $3.60 \pm 0.4 \mathrm{~kg}$ ) was purchased from the Shanghai Laboratory Animal Center (Shanghai, China). The rabbits 
were randomly divided into three groups: Control, sham and SSS ( $n=16$ per group). Rabbits in the sham and SSS groups received a high-lipid diet (1\% cholesterol, 5\% yolk, 5\% lard and $89 \%$ basic feedstuff) for 3 months. The disease model was established according to the method described by Zhang et al (9). Briefly, the rabbits were anesthetized with 3\% pentobarbital sodium $(1 \mathrm{ml} / \mathrm{kg})$, administered intravenously. The SA was exposed and ligated proximal to the origin of the VA. Sham-operated rabbits underwent all surgical procedures, with the exception of the SA occlusion.

Eyeblink conditioning procedures. The eyeblink experiment was conducted 30 days after the surgery. Rabbits $(n=10$ per group) were adapted to a Plexiglas restrainer box (Sigma-Aldrich, St. Louis, MO, USA) for a single 20-min session 3 days prior to the initiation of the experiments. The conditioned stimulus (CS) was a $1-\mathrm{kHz}, 85-\mathrm{dB}$ tone, and the unconditioned stimulus (US) was a 6-psi corneal airpuff. In the delay-conditioning paradigm, the duration of the tone was $500 \mathrm{msec}$, followed $400 \mathrm{msec}$ after its onset by a $100-\mathrm{msec}$ airpuff US. The CS and US co-terminated. The rabbits received 7 training sessions over 7 consecutive days. Each session consisted of 80 paired CS-US trials, and the inter-trial interval was 20-30 sec. One week after the last day of training, the rabbits received 15 trials in which only the CS was presented.

5-Bromo-2'-deoxyuridine (BrdU) labeling and tissue preparation. A further 18 rabbits ( $\mathrm{n}=6$ per group) were administered BrdU (Sigma-Aldrich) at a dosage of $100 \mathrm{mg} / \mathrm{kg}$ body weight (one intraperitoneal administration per day) for the last 3 days of the experimental period. The rabbits were sacrificed $2 \mathrm{~h}$ after the last injection. The rabbits were intravenously anesthetized with $3 \%$ pentobarbital sodium $(1 \mathrm{ml} / \mathrm{kg})$ and sacrificed. The obtained tissues were transcardially perfused with ice-cold $0.9 \% \mathrm{NaCl}$ solution, followed by a freshly prepared solution of $4 \%$ paraformaldehyde in $0.1 \mathrm{M}$ sodium phosphate buffer. The brains were removed and postfixed overnight, cryostat-sectioned $(30 \mu \mathrm{m})$ in series and stored at $-80^{\circ} \mathrm{C}$.

Immunohistology. For BrdU and Ki67 immunohistology, antigen retrieval was performed by incubating the slides in $10 \mathrm{mM}$ sodium citrate buffer $(\mathrm{pH} 6.0)$ at $95-98^{\circ} \mathrm{C}$ for $15 \mathrm{~min}$. After cooling for $1 \mathrm{~h}$, the sections were rinsed with $0.1 \mathrm{M}$ phosphate-buffered saline (PBS) and treated with blocking solution (3\% bovine serum albumin, 5\% goat serum and 3\% Triton in 0.1 M PBS; Sigma-Aldrich) for $2 \mathrm{~h}$ at room temperature. The slides were incubated with monoclonal mouse anti-rabbit antibodies against BrdU (1:5,000; NA61-100UGCN; EMD Millipore, Billerica, MA, USA) and Ki67 (1:1,000; 12160; Cell Signaling Technology, Inc., Danvers, MA, USA) overnight at $4^{\circ} \mathrm{C}$. After washing three times in 0.1 M PBS with Tween-20, the sections were incubated with the Cyanine 3-conjugated secondary goat anti-mouse Alexa Fluor 488 antibodies (1:1,000; A10547; Invitrogen Life Technologies, Carlsbad, CA, USA) for $2 \mathrm{~h}$ at room temperature. The sections were counterstained with DAPI and mounted with an aqueous mounting medium.

Cerebellar dialysis and high-performance liquid chromatography (HPLC) measurements. Extracellular concentrations of adenosine triphosphate (ATP) and adenosine in the cerebellar cortex were evaluated using microdialysis techniques. The animals were positioned in a stereotactic frame. Prior to insertion, the probes were perfused with artificial cerebrospinal fluid (CSF), containing $147 \mathrm{mM} \mathrm{NaCl}, 4 \mathrm{mM} \mathrm{KCl}, 2.3 \mathrm{mM}$ $\mathrm{CaCl}_{2}$ and $0.9 \mathrm{mM} \mathrm{MgCl}$ (pH 7.4), at a rate of $2 \mu \mathrm{l} / \mathrm{min}$. In all rabbits, a 360-min stabilization period was implemented following the insertion of the microdialysis probe. The dialysate was collected every $15 \mathrm{~min}$ for later analysis. The concentrations of ATP and adenosine were determined using an Agilent 1200 HPLC system with a photodiode array detector (Agilent Technologies, Santa Clara, CA, USA), and separation was performed using a Hypersil base-deactivated silica C-18 reverse-phase column (column length, $300 \mathrm{~mm}$; column ID, $4.6 \mathrm{~mm}$; particle size, $10 \mu \mathrm{m}$; Thermo Scientific, Inc., Waltham, MA, USA). The mobile phase consisted of $0.1 \mathrm{M}$ phosphate buffer ( $\mathrm{pH} 7.0)$ and methanol (1\%). The flow rate was $1 \mathrm{ml} / \mathrm{min}$ and the column temperature was $25^{\circ} \mathrm{C}$. Ultraviolet (UV) detection was conducted at $254 \mathrm{~nm}$ using an Ultraviolet Detector (GD-3; Puyang Medical Instrument Co. Ltd., Nanjing, China). The concentrations of ATP and adenosine were quantified based on linear calibration.

Cytokine and enzyme activity assays. The levels of interleukin (IL)-1 $\beta$, IL-6, malondialdehyde (MDA) and 8-hydroxy-2'-deoxyguanosine (8-OHdG) and the activities of $\mathrm{CuZn-superoxide} \mathrm{dismutase} \mathrm{(CuZn-SOD)} \mathrm{and} \mathrm{catalase} \mathrm{(CAT)}$ were determined using commercial kits (Nanjing Jiancheng Bioengineering Research Institute, Nanjing, China).

Statistical analysis. Data are expressed as the mean \pm standard error of the mean. The differences in cell-counting results were analyzed using the non-parametric Mann-Whitney test. Repeated measures analysis of variance (ANOVA) and Fisher's protected least significant difference (LSD) multiple comparison test were used to determine differences in ATP and adenosine concentrations among the groups. All other results were compared using one-way ANOVA, followed by the LSD post hoc test for intergroup comparisons. $\mathrm{P}<0.05$ was considered to indicate a statistically significant difference.

\section{Results}

Behavioral experiments. To observe the effects of SSS on the cognition of the rabbits, eyeblink experiments were conducted. No significant differences were found among the results from three groups (Fig. 1; P>0.05).

Effects of SSS on newly generated cells in the rabbit brains. Neurogenesis in the rabbit brains was examined. Quantitative assessment revealed no significant difference in the number of BrdU- or Ki67-labeled cells in the subventricular or subgranular zones among the three groups (data not shown). Notably, a reduced number of BrdU- and Ki67-positive cells were observed in the cerebellar cortex of rabbits in the SSS group $\left(101 \pm 21\right.$ and $85 \pm 17$ cells $/ \mathrm{mm}^{2}$, respectively) compared with the control (178 \pm 33 and $146 \pm 25$ cells $/ \mathrm{mm}^{2}$, respectively) and sham $\left(198 \pm 19\right.$ and $158 \pm 21$ cells $/ \mathrm{mm}^{2}$, respectively) groups (Fig. 2; $\mathrm{P}<0.05$ ), suggesting that SSS exerted a negative impact on neurogenesis in the cerebellar cortex. 


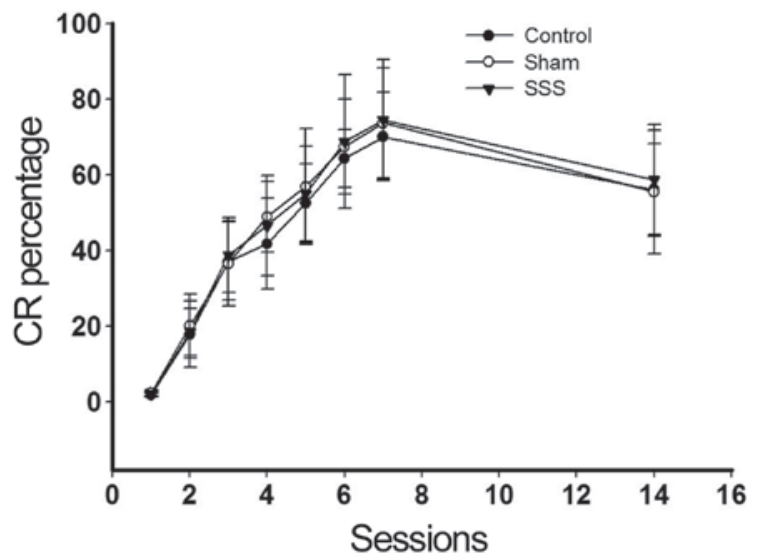

Figure 1. Mean percentage of CR for the 8 sessions of eyeblink classical conditioning. No significant difference was detected among the three groups ( $\mathrm{P}>0.05)$. SSS, subclavian steal syndrome; $\mathrm{CR}$, conditioned responding.
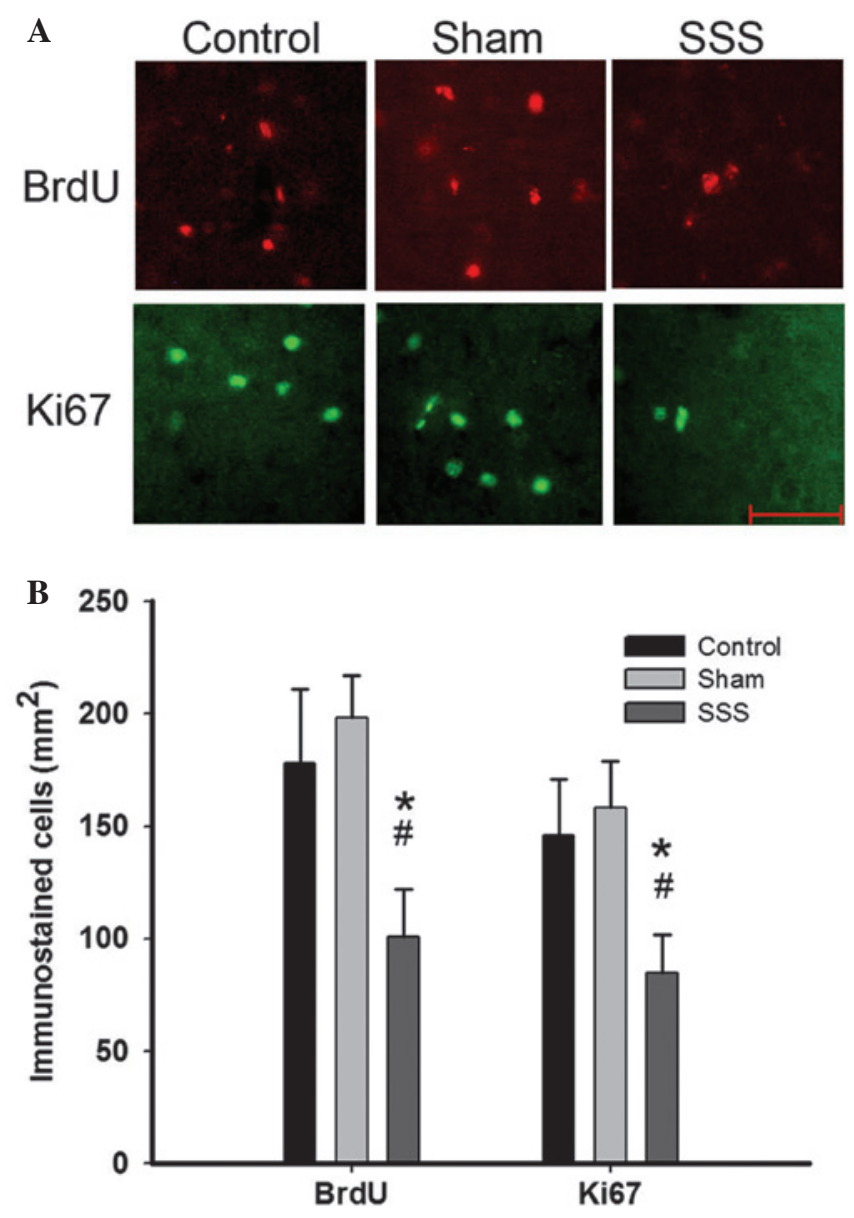

Figure 2. BrdU- and Ki67-immunostained cells in the cerebellar cortex of rabbits. (A) BrdU and Ki67 immunhistology results from representative slices of the control, sham and SSS groups (scale bar, $50 \mu \mathrm{m}$ ). (B) Statistical results of neurogenesis in the cerebellar cortex of rabbits. Data are expressed as the mean \pm standard error of the mean. ${ }^{*} \mathrm{P}<0.05$ vs. sham group; ${ }^{*} \mathrm{P}<0.05$ vs. control group. SSS, subclavian steal syndrome; BrdU, 5-bromo-2'-deoxyuridine.

Extracellular concentrations of ATP and adenosine in the cerebellar cortex. ATP and adenosine levels in the CSF of the cerebellar cortex were measured in vivo using microdialysis. The CSF samples were processed using HPLC, and the levels of ATP and adenosine were determined with a UV

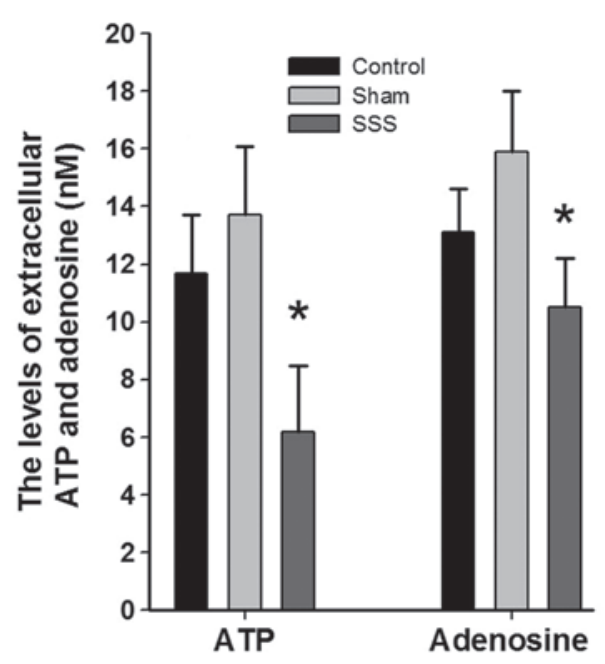

Figure 3. Levels of extracellular ATP and adenosine in the cerebellar cortex of rabbits. Data are expressed as the mean \pm standard error of the mean. ${ }^{*} \mathrm{P}<0.05$ vs. sham group. SSS, subclavian steal syndrome; ATP, adenosine triphosphate.

detector. Immediately following probe insertion, the ATP and adenosine levels were elevated due to the tissue damage caused by probe insertion. The initially elevated levels of ATP and adenosine declined to plateau levels within $6 \mathrm{~h}$. At $6 \mathrm{~h}$ after probe insertion, the extracellular concentrations of ATP and adenosine in the SSS group rabbits $(n=5)$ were $6.2 \pm 2.3$ and $10.5 \pm 1.7 \mathrm{nM}$ compared with the control group, respectively (Fig. 3; $\mathrm{P}<0.05$ ). By comparison, the concentrations of ATP and adenosine in the control and sham group rabbits ( $\mathrm{n}=5$ per group) were $11.7 \pm 2.0$ and $13.1 \pm 1.5 \mathrm{nM}$, and $13.7 \pm 2.4$ and $15.9 \pm 2.1 \mathrm{nM}$, respectively (Fig. 3). Thus, reduced levels of ATP and decreased levels of adenosine were found in the cerebellar cortex, and these submicromolar differences in ATP concentration among the groups were consistent with the results of the immunohistological analyses.

SSS does not affect cytokine levels. No differences were detected in the levels of IL-1 $\beta$, IL-6, MDA, 8-OHdG, CuZn-SOD and CAT among the three groups (Fig. 4; P>0.05).

\section{Discussion}

Patients with SSS may present with a series of neurological and vascular symptoms; however, the condition is most commonly diagnosed as an incidental finding in asymptomatic patients. For certain patients, the symptoms of cerebral ischemia resulting from SSS may be aggravated by arm exercise. Reduced flow resistance due to exercise-induced vasodilation in the upper extremities may cause the diversion of cerebral blood flow to threshold in certain patients, resulting in an increased risk of ischemic stroke in the posterior circulation (8). As the brainstem and cerebellum are primarily supplied by the basilar and vertebral arteries, ischemic stroke in the posterior circulation can result in a broad range of symptoms. These include classic manifestations of ischemia in the posterior territory, including reduction in consciousness, visual field defects, vertigo, ataxia and eye movement disorders, in addition to non-localizing symptoms, such as confusion, disorientation and memory loss (10). 
A

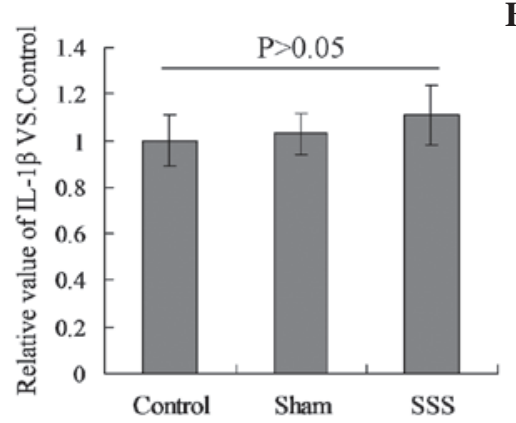

D

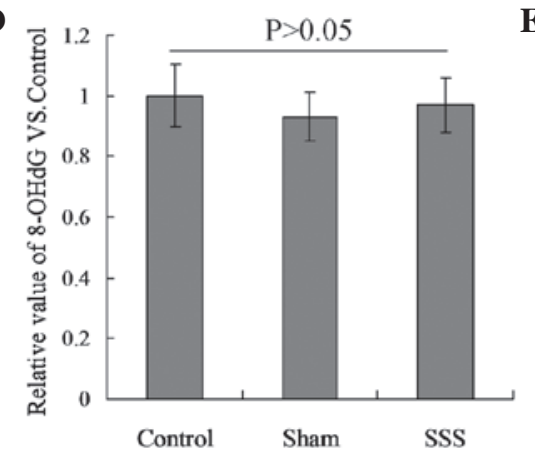

B

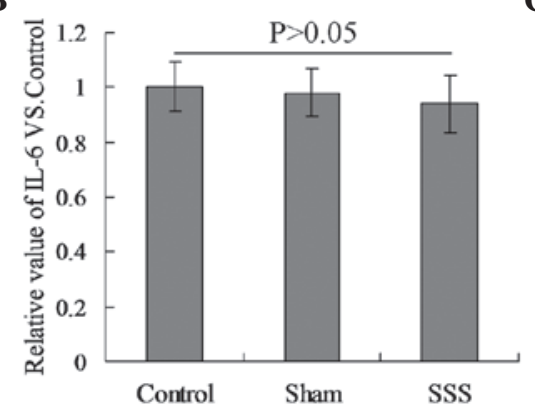

$\mathbf{E}$

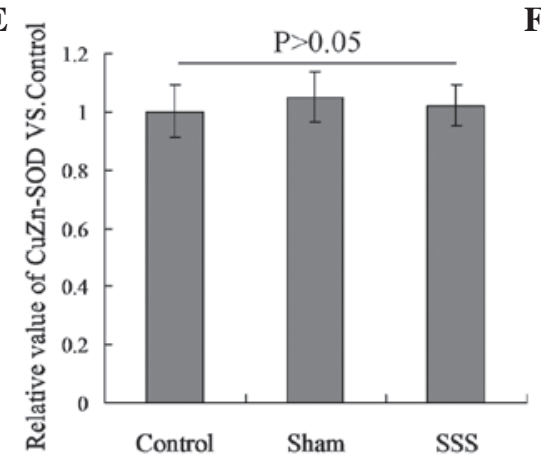

C

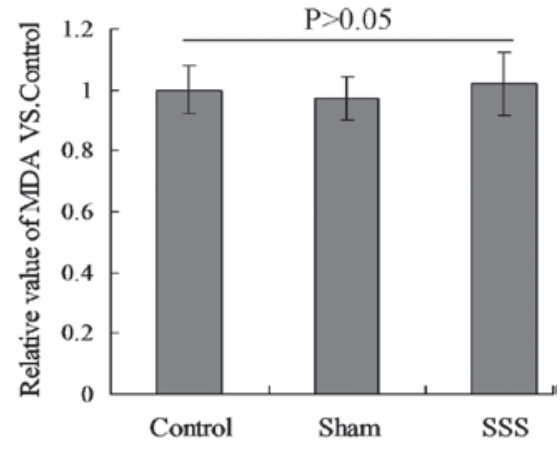

$\mathbf{F}$

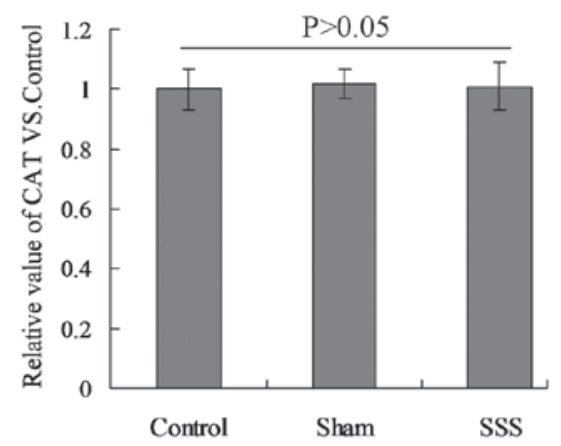

Figure 4. Relative values of (A) IL-1 $\beta$, (B) IL-6, (C) MDA, (D) 8-OHdG, (E) CuZn-SOD and (F) CAT activity in the three groups. IL, interleukin; SSS, subclavian steal syndrome; MDA, malondialdehyde; 8-OHdG, 8-hydroxy-2'-deoxyguanosine; CuZn-superoxide dismutase; CAT, catalase.

The cerebellum plays a central role in movement execution and motor control functions, such as maintaining balance and muscle tension. Furthermore, it has been suggested that the cerebellum is involved in learning and memory, cognition, speech, perception and the generation of emotions (11). In the present study, the effects of SSS on the cognition of rabbits were characterized using eyeblink experiments. Although the results of the eyeblink experiments indicated no significant difference among the three groups, SSS appeared to exert a negative impact on neurogenesis in the rabbit cerebellar cortex (Fig. 2; $\mathrm{P}<0.05$ ).

A previous study described the existence of a secondary germinal matrix persisting after puberty in a subpial position of the cerebellum, called the subpial layer (SPL), in the New Zealand white rabbit (12). The SPL originates from structural modifications of the external granule layer and has the ability to generate neuronal precursors on the cerebellar surface. Analyses of neurogenesis in the cerebellum at different stages, by the detection of Ki67 and BrdU, revealed marked cell proliferation occurring around puberty (12). In addition, it was notable that a number of newly born cells were detectable in fully adult rabbits (1-2 years old) in the absence of a germinative SPL. The function of these newly generated cells is not yet clear, although it is possible that these new cells (such as interneurons) are required in neuronal circuits formed by pre-existing cells (13). The neural circuits in the cerebellar cortex are simple, and the function of the cerebellar cortex is closely associated with motor learning (14). Wang and Liu (15) investigated the motor learning process in rabbits using an eyeblink experiment. The results showed that the cerebellar cortex was able to execute the function of spatial and temporal focusing in motor learning and control. On the basis of these results and those of previous studies, it is possible that the negative effect of SSS on neurogenesis observed in the present study may potentially result in the damage of motor memory.

In order to further clarify the mechanisms underlying the SSS-induced reduction in cell proliferation, the energy metabolism (extracellular ATP and adenosine), immune function (IL-1 $\beta$ and IL-6) and oxidative stress (CuZn-SOD, CAT, MDA and $8-\mathrm{OHdG}$ ) statuses were detected. The results indicated that energy metabolism in the cerebellar cortex was altered, while the levels of extracellular ATP were decreased and the levels of adenosine were also decreased (Fig. 3; P<0.05). It is established that brain tissues respire glucose to produce energy under normal conditions. While under the conditions of chronic ischemia, this glucose utilization in the cerebellum is reduced. We hypothesized that chronic ischemia induced by SSS were the cause of the observed changes. Extracellular ATP is involved in the induction of neurogenesis (16). Deficient ATP release from astrocytes leads to dysfunction in neural stem cell proliferation, and this can be normalized by the administration of exogenous ATP (17). Furthermore, it has been revealed that ATP functions as a mitogenic signal for progenitor and neural stem cells, potentially in an autocrine manner (16). In addition, previous studies have demonstrated that cognitive deficits induced by seizures in young animals may be a consequence of the increased ATP hydrolysis $(18,19)$. In the present study, SSS appeared to induce a reduction in extracellular ATP levels in rabbits, and we hypothesize that this change may impair cognition in rabbits, via direct and indirect mechanisms.

In conclusion, the results of the present study suggest that SSS may inhibit neurogenesis in the cerebellar cortex of rabbits. Furthermore, the findings indicate that the extracel- 
lular ATP reduction may be closely associated with reduced cell proliferation. Collectively, the results of the present study suggest that SSS may impair cognitive function in rabbits. The early diagnosis and treatment of SSS may, therefore, mitigate or prevent cognitive impairment in the future.

\section{Acknowledgements}

This study was supported by the National Key Clinical Unit Project (2011).

\section{References}

1. Reivich M, Holling HE, Roberts B And Toole JF: Reversal of blood flow through the vertebral artery and its effect on cerebral circulation. N Engl J Med 265: 878-885, 1961.

2. Aribas BK, Arda K, Aribas O, Ciledag N, Yologlu Z, Aktas E, Seber T, Kavak S, Cosar Y, Kaygusuz H and Tekin E: Comparison of subcutaneous central venous port via jugular and subclavian access in 347 patients at a single center. Exp Ther Med 4: 675-680, 2012

3. Fields WS: Reflections on 'the subclavian steal'. Stroke 1: 320-324, 1970 .

4. Smith JM, Koury HI, Hafner CD and Welling RE: Subclavian steal syndrome. A review of 59 consecutive cases. J Cardiovasc Surg (Torino) 35: 11-14, 1994.

5. de Souza JM, Espinosa G, Santos Machado M and Soares PJ Bilateral occlusion associated to steal phenomenon of internal carotid and left subclavian arteries: treatment by angioplasty and stenting. Surg Neurol 67: 298-302, 2007.

6. Alcocer F, David M, Goodman R, Jain SK and David S: A forgotten vascular disease with important clinical implications. Subclavian steal syndrome. Am J Case Rep 14: 58-62, 2013.

7. Sharma VK, Chuah B, Teoh HL, Chan BP, Sinha AK and Robless PA: Chronic brainstem ischemia in subclavian steal syndrome. J Clin Neurosci 17: 1339-1341, 2010.
8. Yonas H, Smith HA, Durham SR, Pentheny SL and Johnson DW: Increased stroke risk predicted by compromised cerebral blood flow reactivity. J Neurosurg 79: 483-489, 1993.

9. Zhang GY, Chen ZQ, Ling F, Li YJ, Wang Y and Gu BX: Establishment of a novel hemodynamic cerebral ischemia model of atherosclerotic rabbit. Neurol India 58: 191-194, 2010.

10. Markus HS, van der Worp HB and Rothwell PM: Posterior circulation ischaemic stroke and transient ischaemic attack: Diagnosis, investigation and secondary prevention. Lancet Neurol 12: 989-998, 2013.

11. Buckner RL: The cerebellum and cognitive function: 25 years of insight from anatomy and neuroimaging. Neuron 80: 807-815, 2013.

12. Ponti G, Peretto P and Bonfanti L: A subpial, transitory germinal zone forms chains of neuronal precursors in the rabbit cerebellum. Dev Biol 294: 168-180, 2006.

13. Ponti G, Crociara P, Armentano M and Bonfanti L: Adult neurogenesis without germinal layers: The 'atypical' cerebellum of rabbits. Arch Ital Biol 148: 147-158, 2010.

14. Yoshizawa K, Emoto Y, Kinoshita Y, Yuri T and Tsubura A: $\mathrm{N}$-methyl-N-induced cerebellar hypoplasia in rats: Effect of arachidonic acid supplementation during the gestational, lactational and post-weaning periods. Exp Ther Med 6: 627-634, 2013.

15. Wang L and Liu SQ: Neural circuit and its functional roles in cerebellar cortex. Neurosci Bull 27: 173-184, 2011.

16. Lin JH, Takano T, Arcuino G, Wang X, Hu F, Darzynkiewicz Z, Nunes M, Goldman SA and Nedergaard M: Purinergic signaling regulates neural progenitor cell expansion and neurogenesis. Dev Biol 302: 356-366, 2007.

17. Cao X, Li LP, Qin XH, Li SH, Zhang M, Wang Q, Hu HH, Fang YY, Gao YB, Li XW, et al: Astrocytic adenosine 5'-triphosphate release regulates the proliferation of neural stem cells in the adult hippocampus. Stem Cells 31: 1633-1643, 2013.

18. Cognato GP, Vuaden FC, Savio LE, Bellaver B, Casali E, Boqo MR, Souza DO, Sevigny J and Bonan CD: Nucleoside triphosphate diphosphohydrolases role in the pathophysiology of cognitive impairment induced by seizure in early age Neuroscience 180: 191-200, 2011

19. Lu L, Tang L, Wei W, Hong Y, Chen H, Ying W and Chen S: Nicotinamide mononucleotide improves energy activity and survival rate in an in vitro model of Parkinson's disease. Exp Ther Med 8: 943-950, 2014 